\begin{tabular}{|c|l|}
\hline Title & $\begin{array}{l}\text { Functional Significance of the Preconditioning-induced Down-regul ation of Glutamate Transporter GLT-1 in } \\
\text { Neuron/astrocyte Co-cultures }\end{array}$ \\
\hline Author(s) & Kosugi, Tatsuro; Kawahara, Koichi; Y amada, Takeshi; Nakajima, Takay uki; Tanaka, Motoki \\
\hline Citation & $\begin{array}{l}\text { Neurochemical Research, 30(9), 1109-1116 } \\
\text { https://doi.org/10.1007/311064-005-8219-z }\end{array}$ \\
\hline Issue Date & $2005-09$ \\
\hline Doc URL & http://hdl.handle.net/2115/5971 \\
\hline Rights & The original publication is available at www.springerlink.com \\
\hline Type & article (author version) \\
\hline File Information & NR30-9.pdf \\
\hline
\end{tabular}

Instructions for use 


\section{Functional significance of the preconditioning-induced down-regulation of glutamate transporter GLT-1 in neuron/astrocyte co-cultures.}

Tatsuro Kosugi, Koichi Kawahara, Takeshi Yamada, Takayuki Nakajima, and Motoki
Tanaka

Laboratory of Cellular Cybernetics, Graduate School of Information Science and

Technology, Hokkaido University, Sapporo 060-0814, Japan

Running head: preconditioning-induced down-regulation of GLT-1

Corresponding to:

Koichi Kawahara, Ph D.

Professor of the Laboratory of Cellular Cybernetics,

Graduate School of Information Science and Technology,

Hokkaido University, Sapporo 060-0814

Japan.

E-mail: kawahara@cellc.ist.hokudai.ac.jp 


\begin{abstract}
In the brain, prior sublethal ischemia (preconditioning, PC) is known to produce tolerance of neurons to subsequent lethal ischemia. This study aims at elucidating what alterations were induced in neurons and/or astrocytes by PC treatment. The rise in the extracellular concentration of glutamate during ischemia was markedly suppressed by the prior PC treatment. Immunocytochemical and Western blot analyses demonstrated that the expression of the astrocytic glutamate transporter GLT-1 was transiently down-regulated after the PC insult. The PC insult possibly suppressed the neuron-derived factors up-regulating GLT-1. Here we show that PC-induced down-regulation of GLT-1 is crucial for the increased neuronal resistance to subsequent severe ischemic insult.
\end{abstract}

Key words GLT-1, preconditioning, ischemic tolerance, neuron-derived factor 


\section{Introduction}

Preconditioning (PC) to ischemic tolerance is a phenomenon in which a brief subtoxic insult induces robust protection against the deleterious effects of a subsequent, prolonged, lethal ischemia (1). In the brain, Kitagawa et al. (2) first reported that gerbils subjected to a sublethal transient global ischemia exhibited reduced hippocampal neuronal death after a severe ischemic insult 24-28 h later. Although numerous studies have been performed on this phenomenon, this mechanism has not been fully delineated at present.

Extracellular concentration of L-glutamate (Glu) in the mammalian brain is subjected to homeostasis because an elevated concentration of Glu results in the excessive activation of neuronal Glu receptors, especially NMDA-type receptors, thereby resulting in massive neuronal death (3). Previous studies including by us have demonstrated that the astrocytic Glu transporter GLT-1 is primarily responsible for the clearance of extracellular Glu (4-7). These findings have led to an idea that the normal function of astrocytic Glu transporter is crucial to the survival of neurons in the brain. Under brain ischemia, however, the role of astrocytic Glu transporters is reversed; that 
is, Glu is released from astrocyte to the extracellular space $(8,9)$, resulting in massive excitotoxic death of neurons.

We have recently demonstrated that the reversed operation of Glu transporter GLT-1 during subtoxic PC insult is necessary for the development of PC-induced ischemic tolerance of neurons (10). This finding has suggested for the first time that the change in glial function during prior PC is critical to the development of neuronal ischemic tolerance. However, the question remains unanswered as to what alterations are induced in the neuronal and/or glial function 24-48 h after the PC insult. Here we show that the expression of GLT-1 was transiently down-regulated after the PC insult, and that neurons were possibly involved in such PC-induced down-regulation. 


\section{Experimental procedure}

The animal experiments conformed to the "Principles of laboratory animal care" (NIH publication No. 85-23, revised 1996), as well as the "guide for the care and use of laboratory animals”, Hokkaido University School of Medicine.

\section{Neuron/astrocyte co-culture}

Culture methods were described previously in detail $(4,10,11)$. In brief, neurons/astrocytes were prepared from 17-day old embryonic rat cortices and grown in Dulbecco’s modified Eagle’s medium (DMEM, Gibco, Grand Island, NY) which was supplemented with 10\% heat-inactivated fetal bovine serum (FBS), 10\% Ham's F12, and $0.24 \%$ penicillin/streptomycin (culture medium) and maintained in a $5 \% \mathrm{CO}_{2}$ incubator at $37^{\circ} \mathrm{C}$. The cultures were fed a conditioned medium (CM) twice a week. To obtain the CM, cells from the 17-day old embryonic rat cortices were plated onto poly-L-lysine-coated 6-well dishes and cultured for more than 2 weeks. The cultures were then fed a cooled culture medium and incubated for an additional day. After 13-15 days, the neurons in these cultures sit on the top of a confluent monolayer of astrocytes. The experiments were performed using these cultures.

\section{Astrocyte-enriched culture}


The cultures without neurons were prepared from primary neuron/astrocyte co-cultures according to the method of Nakanishi et al (12). After 7 days in the primary culture, cultured glial components were dislodged enzymatically ( $0.1 \%$ trypsin), and plated onto other dishes. The cultures were fed a CM twice a week similar to the co-cultures. After 14-15 days, the astrocytes in these cultures become a confluent monolayer.

\section{Immunocytochemistry}

Astrocytes and neurons were identified by immunostaining with antibodies against glial fibrillary acidic protein (GFAP; Sigma, St Louis, MO, 1:1000) and microtubule-associated protein 2 (MAP-2; Sigma, 1:400), respectively. The astrocytic glutamate transporter GLT-1 was detected by immunostaining with anti-GLT-1 polyclonal antibodies (Chemicon, Temecula, CA. 1:2500). For labeling, a 1:500 dilution of biotinylated goat antibody against mouse or guiniea pig IgG (Vector Laboratories, Burlingame, CA) was used. Bound antibodies were detected by the avidin-biotin-peroxidase complex (ABC) method, using a commercial ABC kit (Vector Laboratories). Observation of the peroxidase activity was made possible by incubation with $0.1 \%$ 3,3'-diaminobenzidine tetrahydrochloride (DAB) in a $50 \mathrm{mM}$ Tris- $\mathrm{HCl}$ buffer (pH 7.4) supplemented with $0.02 \% \mathrm{H}_{2} \mathrm{O}_{2}$. 


\section{Western blot analysis}

Cell lysates were diluted 3:1 in sample buffer (187.5 mM Tris-HCl containing $6 \%$ SDS, and 15\% glycerol, 15\% 2-mercaptoethanol) and denatured at $100{ }^{\circ} \mathrm{C}$ for $3 \mathrm{~min}$. Proteins were electrophoresed on a 10\% SDS-polyacrylamide gel and transferred onto a polyvinylidene difluoride membrane (Bio-Rad, Hercules, CA). Nonspecific binding sites were blocked with 5\% nonfat milk for $60 \mathrm{~min}$, and then the membrane was incubated overnight at $4{ }^{\circ} \mathrm{C}$ with one of the following antibodies: polyclonal antibody to GLT-1 (1:10000), GLAST (1:8000) (Chemicon), and monoclonal antibody to actin (1:2000) (Sigma), and then horseradish peroxidase-labeled anti-guinea pig (1:2000) (Cell Signaling Technology) or anti- mouse IgG antibody for actin (1:2000) (Cell Signaling Technology), respectively. The immunoreactive bands were detected with an enhanced chemiluminescence kit (NEN Life Science Products, Boston, MA). Quantification of the levels was preformed by densitometric analysis using Scion Image Beta 4.02 (Scion Corporation).

\section{Oxygen-Glucose deprivation}

Cortical cultures were subjected to oxygen-glucose deprivation (OGD) injury using a protocol described previously $(10,11,13)$. In brief, cultures were placed in an anaerobic chamber containing the deoxygenation reagent and glucose-free balanced salt 
solution (BSS: $116 \mathrm{mM} \mathrm{NaCl}, 0.8 \mathrm{mM} \mathrm{MgSO}_{4}, 5.4 \mathrm{mM} \mathrm{KCl}, 1.0 \mathrm{mM} \mathrm{NaH}{ }_{2} \mathrm{PO}_{4}, 26.2$ $\mathrm{mM} \mathrm{NaHCO} 3,1.8 \mathrm{mM} \mathrm{CaCl}_{2}$, and $0.01 \mathrm{mM}$ glycine) was added. Near anoxic conditions were achieved using an Anaero-Pack System (Mitsubishi Gas Chemical, Tokyo, Japan). To terminate OGD, cultures were carefully washed with glucose (20 $\mathrm{mM}$ ) containing BSS, and then incubated again in culture medium at $37^{\circ} \mathrm{C}$ in $95 \%$ air-5\% $\mathrm{CO}_{2}$ (reperfusion). Cultures with sham treatment not deprived of oxygen and glucose were placed in BSS containing $20 \mathrm{mM}$ glucose.

\section{Measurement of the extracellular glutamate concentration}

The extracellular concentration of glutamate (Glu) was measured using an enzymatic assay $(14,15)$. In the presence of Glu and $\beta$-nicotinamide adenine dinucleotide (NAD ${ }^{+}$), L-glutamic dehydrogenase (GDH) produces $\alpha$-ketoglutarate and $\mathrm{NADH}$, a product that fluoresces when excited at $360 \mathrm{~nm}$. In the presence of $\alpha$-ketoglutarate and L-Alanine, glutamate pyruvate transaminase (GPT) produces L-glutamate. Therefore, GDH (50 U/mL), NAD ${ }^{+}$(2 mM), GPT (4 U/mL), and L-Alanine (2 mM) were added to the external solution, and the fluorescence was excited at $360 \mathrm{~nm}$ and detected at $>510 \mathrm{~nm}$ with a fluorescent microscope. Images excited at $360 \mathrm{~nm}$ were acquired with integration times of $5 \mathrm{~s}$ at intervals of $20 \mathrm{~s}$. The extracellular Glu was detected as an increase in NADH fluorescence. This method 
does not necessarily reflect the quantitative extracellular Glu level, but can detect subtle changes in the concentration of Glu.

\section{Survival rate of neurons}

Neuronal death was analyzed following observation of the nuclear morphology using the fluorescent DNA-binding dyes, Hoechst 33342 (H33342) and propidium

iodide (PI). Individual nuclei were observed using fluorescent microscopy (Olympus, IX70, Tokyo, Japan) and subsequently analyzed. PI was used to identify nonviable cells. More specifically, an average of 450-500 neurons from random fields were analyzed in each experiment. The survival rate of neurons - meaning the percentage of viable neurons remaining - was determined by placing images of nuclear staining on phase-contrast images, and calculating (viable neurons/total neurons before drug treatment $) \times 100$. At least 4 independent experiments $(n \geq 4)$ were conducted and analyzed.

\section{NCM, PC-NCM treatment}

To obtain neuron-conditioned medium (NCM) or preconditioned NCM (PC-NCM), we incubated neuron-glia co-cultures on day 14 of cultivation, and sham or PC treatment was conducted, respectively. At $24 \mathrm{~h}$ after the treatment, the medium 
was collected and centrifuged at $3000 \times \mathrm{g}$ to remove cells and membrane fragments.

Thereafter, the conditioned media were fed to astrocyte-enriched cultures for $24 \mathrm{~h}$.

\section{Statistics}

The data are expressed as the mean \pm S.D. Comparisons were performed using the one-way analysis of variance (ANOVA) followed by a paired t-Test. A $P$ value of less than 0.01 or 0.05 was considered statistically significant. 


\section{Results}

We first confirmed whether preconditioning (PC)-induced neuronal ischemic tolerance in murine cortical cultures containing both neurons and glia (from E17 Wistar rats and days in vitro 13-16) was a transient phenomenon, and delayed neuronal death after ischemia was induced by exposure to glutamate. Brain ischemia was simulated by depriving the cultures of both oxygen and glucose (OGD) $(10,11,13)$. Cultures were preconditioned with a 60 min OGD and then exposed to a lethal (90 min) OGD 24 and $72 \mathrm{~h}$ later (Fig. 1A). Exposure of preconditioned cultures to the lethal OGD $24 \mathrm{~h}$ later resulted in a significant increase in the survival rate of neurons inspected $24 \mathrm{~h}$ after the end of the lethal insult. However, no significant protective effect on neuronal death was observed in the preconditioned cultures exposed to the lethal insult $72 \mathrm{~h}$ later, indicating that the PC-induced neuronal ischemic tolerance was a transient phenomenon. Suppression of the activation of NMDA-type glutamate receptors during the lethal 90 min OGD by treatment with DL-2-amino-5-phosphonopentanoic acid (AP5, $100 \mu \mathrm{M}$ ), a specific inhibitor of NMDA receptors, resulted in a significant increase in the survival rate of neurons (Fig. 1A). This result suggested that OGD-induced neuronal death was caused by the increased concentration of extracellular glutamate resulting in the excessive activation of NMDA receptors. If this was the case, PC treatment in 
advance was expected to increase the neuronal resistance to glutamate-mediated excitotoxicity. Thus, we next investigated whether the prior PC treatment increased the resistance of neurons to glutamate.

Interestingly, neurons in the preconditioned co-cultures showed decreased tolerance of glutamate toxicity. Treatment of co-cultures with glutamate (65 and 75 $\mu \mathrm{M}$ ) for 20 min did not result in the significant death of neurons both in the sham-treated and preconditioned co-cultures (Fig. 1D). In contrast, treatment with 85 $\mu \mathrm{M}$ glutamate for 20 min did not induce massive neuronal death in the sham-treated co-cultures (Fig. 1B and D), but treatment $24 \mathrm{~h}$ after the PC insult with the same concentration of glutamate for 20 min resulted in a significant decrease in the survival rate of neurons $24 \mathrm{~h}$ later (Fig. 1C and D). These results suggested that the PC insult did not increase the neuronal tolerance to glutamate toxicity, but possibly reduced the rise in the extracellular concentration of glutamate during OGD.

We therefore investigated whether the rise in the extracellular concentration of glutamate during OGD was being suppressed after the PC treatment. For the measurement of extracellular glutamate, glutamate was changed to NADH, a product that fluoresces when excited at $360 \mathrm{~nm}$ in an enzymatic assay, and the concentration of glutamate was estimated as the increase in NADH fluorescence (See Method) $(14,15)$. 
In the cultures preconditioned $24 \mathrm{~h}$ before, the OGD-induced rise in the extracellular concentration of glutamate was significantly suppressed as compared with that in the cultures without preconditioning (sham) (Fig. 2A, B, and D). We have recently revealed that the reversed operation of the astrocytic glutamate transporter GLT-1 is crucially involved in the marked elevation of glutamate during $\operatorname{OGD}(4,10)$. When the cultures were treated with DHK (200 $\mu \mathrm{M})$, a selective blocker of astrocytic GLT-1 $(5,16,17)$, the OGD-induced rise of extracellular glutamate was significantly suppressed similar to in the preconditioned cultures (Fig. 2C and D), suggesting that the expression of astrocytic GLT-1 was down-regulated by the PC insult. We next tested this possibility.

We investigated whether the PC insult changed the expression of the astrocytic glutamate transporters GLT-1 and GLAST by conducting immunocytochemical as well as Western blot analyses (see Methods). At 24 and $72 \mathrm{~h}$ after the PC insult, there was no difference in GLAST and actin expression levels (Fig. 3A and B). In contrast, the expression of astrocytic GLT-1 was significantly decreased $24 \mathrm{~h}$ after the PC, but recovered to the control level at $72 \mathrm{~h}$ (Fig. 3A, B, C, and D). A quantitative analysis showed that the expression of GLT-1 in cultures $24 \mathrm{~h}$ after the PC treatment decreased to about $60 \%$ of the level in sham-treated cultures. These results suggested that the 
expression of GLT-1 was transiently down-regulated $24 \mathrm{~h}$ after the PC insult, contributing to the decreased rise in the concentration of extracellular glutamate via the reversed operation of GLT-1 during ischemia. However, the decreased expression of astrocytic GLT-1 caused by the PC insult may impair the astrocytic clearance of extracellular glutamate. This was the reason why neurons in the preconditioned neuron/astrocyte co-cultures showed the increased vulnerability to glutamate-mediated toxicity observed in Fig. 1.

We finally investigated the possibility that neurons in neuron/astrocyte co-cultures were involved in the regulation of this PC-induced temporal decrease in GLT-1 expression. Previous studies have revealed that the expression level of GLT-1 is regulated by several factors like epidermal growth factor (EGF) or transforming growth factor- $\alpha$ (TGF- $\alpha$ ) in cultured astrocytes (18). In addition, pituitary adenylate cyclase-activating polypeptide (PACAP), a neuron-derived peptide, has been identified as crucial for up-regulating GLT-1 expression when glial cultures are treated with neuron-conditioned medium (NCM) (19). These findings seem to support the immunohistochemical finding that GLT-1-positive astrocytes were preferentially identified in astrocytes just underneath the aggregate of neurons (Fig. 3E1). We therefore investigated whether the PC insult $24 \mathrm{~h}$ prior decreased the neuronal release of 
such factors up-regulating the expression of astrocytic GLT-1. To do this, the changes in the expression of GLT-1 were analyzed in astrocyte-enriched cultures treated with the conditioned medium from either the sham-treated or the preconditioned neuron/astrocyte co-cultures (NCM, PC-NCM) for 24 h. Astrocyte-enriched cultures were prepared by the treatment of neuron/astrocyte co-cultures with trypsin (see Methods) (12). An immunocytochemical analysis using anti-microtuble-associated protein-2 (MAP-2) antibody showed that MAP2-positive neurons were not present (Fig. 4B2), and almost all the cells were stained by an anti-glial fibrillary acidic protein (GFAP) antibody in our astrocyte-enriched cultures (Fig. 4B3). Trypsin did not affect the density of GFAP-positive astrocytes. In the astrocyte-enriched cultures, few GLT-1-positive astrocytes were observed (Fig. 4B4). For preparing the NCM, we incubated neuron/astrocyte co-cultures at 14 days in vitro in conditioned-medium (See Method). After $24 \mathrm{~h}$, the medium was collected and used as the NCM for astrocyte-enriched cultures. Treatment of astrocyte-enriched cultures with NCM for 24 h significantly increased the expression of GLT-1, but treatment with PC-NCM did not (Fig. 4C and D). A quantitative analysis showed that the GLT-1 expression level in the PC-NCM-treated astrocyte-enriched cultures was low and at about $60 \%$ of the level in the NCM-treated cultures after treatment for $24 \mathrm{~h}$. These results suggested that 
neuron-derived factors responsible for the up-regulation of the expression of astrocytic GLT-1 had decreased in level $24 \mathrm{~h}$ after the PC insult. 


\section{Discussion}

This paper has demonstrated a possibility that neurons transiently decreased the expression of astrocytic glutamate transporter GLT-1 after the prior ischemic preconditioning (PC) and contributed to the PC-induced neuronal resistance to subsequent severe ischemic insult.

Although numerous studies including by us have reported increased tolerance to ischemia following preconditioning of the mammalian brain with OGD, cortical spreading depression (CSD), or middle cerebral artery occlusion (MCAO), the mechanism underlying this neuroprotection is unclear (20-23). Some studies have reported that neurons themselves acquire tolerance against ischemia through preconditioning $(22,23)$. However, in our co-cultures, neurons rather became vulnerable to glutamate-mediated death by the prior PC insult (Fig. 1), suggesting that the PC-induced changes in glial function; that is, the down-regulation of astrocytic GLT-1 expression, seemed critical to the PC-induced ischemic tolerance of neurons. In this study, the prior sublethal OGD (PC) decreased the GLT-1 expression and the amount of extracellular glutamate during subsequent lethal OGD (Fig. 2 and Fig. 3). Previous studies, however, have demonstrated that preconditioning of the gerbil brain did not alter the amount of glutamate in the hippocampal CA1 subfield released during 
subsequent second ischemia (24). CA1 pyramidal neurons are known to have high density of NMDA receptors and are most vulnerable to ischemia (25). Therefore, the duration of sublethal ischemia for PC of the hippocampus must be very short, and is usually only two minutes or so (24). The function of astrocytic glutamate transporter GLT-1 might not be reversed during this short period. In this case, the rise in the amount of extracellular glutamate during PC is probably caused by the increased synaptic release. In support of this idea, previous studies have reported that ischemic stress to the brain or brain cell cultures induces a biphasic increase in the extracellular concentration of Glu $(8,26,27)$. The first phase of the elevation is dependent on $\mathrm{Ca}^{2+}$, and is caused by the release of Glu from nerve endings due to $\mathrm{Ca}^{2+}$-dependent exocytosis. The second phase is caused by the reversed uptake of Glu by neuronal and/or astrocytic Glu transporters. Therefore, PC insult with short duration might not result in the down-regulation of GLT-1, and thus does not significantly decrease the amount of extracellular glutamate during the subsequent lethal ischemia.

We have recently found that the activation of NMDA receptors during PC is necessary for the development of PC-induced ischemic tolerance in neurons, and that this activation is caused by the marked elevation in the extracellular concentration of glutamate resulting from the reversed uptake of glutamate by astrocytic GLT-1 (10). 
This study demonstrated that the prior PC resulted in the down-regulation of the expression of GLT-1, and in the decrease in the subsequent lethal ischemia-induced rise in the extracellular concentration of glutamate (Fig. 2 and Fig. 3). However, under normal conditions, astrocytic GLT-1 plays a crucial role in the clearance of excessive extracellular glutamate, and in the protection of neurons from glutamate-mediated toxicity, suggesting that the PC-induced decrease in the expression of astrocytic GLT-1 is also dangerous to the neurons. Therefore, it seems meaningful for neurons that the PC-induced down-regulation of GLT-1 expression is a transient phenomenon (Fig. 3). 


\section{References}

1. Nangopal, K., Dawson, T.M. and Dawson, V.L. 2002. Critical role for nitric oxide signaling in cardiac and neuronal ischemic preconditioning and tolerance. J. Pharmacol. Exp. Ther. 297:474-478.

2. Kitagawa, K., Matsumoto, M., Tagaya, M., Hara, R., Ueda, H., Niinobe, M., Handa, N., Fukunaga, R., Kimura, K., Mikoshiba, K. and Kamada, T. 1990. “Ischemic tolerance” phenomenon found in the brain. Brain Res. 528:21-24.

3. Choi, D.W. 1998. Glutamate neurotoxicity and diseases of the nervous system. Neuron 1:623-634.

4. Kawahara, K., Hosoya, R., Sato, H., Tanaka, M., Nakajima, T. and Iwabuchi, S. 2002. Selective blockade of astrocytic glutamate transporter GLT-1 with dihydrokainate prevents neuronal death during ouabain treatment of astrocyte/neuron co-cultures. GLIA 40:337-349.

5. Rao, V.L.R., Dogan, A., Todd, K.G., Bowen, K.K., Kim, B-T., Rothstein, J.D. and Dempsey, R.J. 2001. Antisense knockdown of the glial glutamate transporter GLT-1, but not the neuronal glutamate transporter EAAC1, exacerbates transient focal cerebral ischemia-induced neuronal damage in rat brain. J. Neurosci. 21:1876-1883. 
6. Rothstein, J.D., Dykes-Hoberg, M., Pardo, C.A., Bristol, L.A., Jin, L., Kuncl, R.W., Kanai, Y., Hediger, M.A., Wang, Y., Schielke, J.P. and Welty, D.F. 1996. Knockout of glutamate transporters reveals a major role for astro-glial transport in excitotoxicity and clearance of glutamate. Neuron 16:675-686.

7. Tanaka, K., Watase, K., Manabe, T., Yamada, K., Watanabe, M., Takahashi, K., Iwama, H., Hori, S., Takimoto, M. and Wada, K. 1997. Epilepsy and exacerbation of brain injury in mice lacking the glutamate transporter GLT-1. Science 276:1699-1702. 8. Szatkowski, M. and Attwell, D. 1994. Triggering and execution of neuronal death in brain ischaemia: two phases of glutamate release by different mechanisms. Trends Neurosci. 9:359-365.

9. Szatkowski, M., Barbour, B. and Attwell, D. 1990. Non-vesicular release of glutamate from glial cells by reversed electrogenic glutamate uptake. Nature 348:443-446.

10. Kawahara, K., Kosugi, T., Tanaka, M., Nakajima, T. and Yamada, T. 2005. Reversed operation of glutamate transporter GLT-1 is crucial to the development of preconditioning-induced ischemic tolerance of neurons in neuron/astrocyte co-cultures. GLIA 49:349-359. 
11. Kawahara, K., Yanoma, J., Tanaka, M., Nakajima, T. and Kosugi, T. 2004. Nitric oxide produced during ischemia is toxic but crucial to preconditioning-induced ischemic tolerance of neurons in culture. Neurochem. Res. 29:797-804.

12. Nakanishi, K., Okouchi, Y., Ueki, T., Asai, K. and Isobe, I. 1994. Astrocytic contribution to functioning synapse formation estimated by spontaneous neuronal intracellular $\mathrm{Ca}^{2+}$ oscillations. Brain Res. 659:169-178.

13. Kawahara, K, Abe, R., Yamauchi, Y. and Kohashi, M. 2002. Fluctuations of contraction rhythm during simulated ischemia/reperfusion in cultured cardiac myocytes from neonatal rats. Biol. Rhythm Res. 33:339-350.

14. Innocenti, B., Parpura, V. and Haydon, P.G. 2000. Imaging extracellular waves of glutamate during calcium signaling in cultured astrocytes. J. Neurosci. 20:1800-1808. 15. Maguire, G., Simko, H., Weinreb, R.N. and Ayoub, G. 1998. Transport-mediated release of endogenous glutamate in the vertebrate retina. Pflügers Arch. 436:481-484. 16. Levy, L.M., Warr, O. and Attwell, D. 1998. Stoichiometry of the glial glutamate transporter GLT-1 expressed inducibly in a chinese hamster ovary cell line selected for low endogenous $\mathrm{Na}^{+}$-dependent glutamate uptake. J. Neurosci. 18:9620-9628. 17. Robinson, M.B. 1998. The family of sodium-dependent glutamate transporters: a focus on the GLT-1 / EAAT2 subtype. Neurochem. Int. 33:479-491. 
18. Figiel, M., Maucher, T., Rozyczka, J., Bayatti, N. and Engele, J. 2003. Regulation of glial glutamate transporter expression by growth factors. Exp. Neurol. 183:124-135.

19. Figiel, M. and Engele, J. 2000. Pituitary adenylate cyclase-activating polypeptide (PACAP), a neuron-derived peptide regulating glial glutamate transport and metabolism.

J. Neurosci. 20:3596-3605.

20. Douen, G.A., Akiyama, K., Hogan, J.M., Wang, F., Dong, L., Chow, K.A. and Hakim, A. 2000. Preconditioning with cortical spreading depression decreases intraischemic cerebral glutamate levels and down-regulates excitatory amino acid transporters EAAT1 and EAAT2 from rat cerebral cortex plasma membranes. $J$. Neurochem. 75:812-818.

21. Kato, H., Liu, Y., Araki, T. and Kogure, K. 1992. MK-801, but anisomycin, inhibits the induction of tolerance to ischemia in the gerbile hippocampus. Neurosci. Lett. 139:118-121.

22. McLaughlin, B., Hartnett, K.A., Erhardt, J.A., Legos, J.J., White, R.F., Barone, F.C. and Aizenman, E. 2003. Caspase 3 activation is essential for neuroprotection in preconditioning. Proc. Natl. Acad. Sci. U S A 21:715-720.

23. Nakajima, T., Iwabuchi, S., Miyazaki, H., Okuma, M., Inanami, O., Kuwabara, M., Nomura, Y. and Kawahara, K. 2002. Relationship between the activation of CREB and 
ischemic tolerance in the penumbra region of rat cerebral cortex. Neurosci. Lett.

331:13-16.

24. Nakata, N., Kato, H., Liu, Y. and Kogure, K. 1992. Effects of pre-treatment with

sublethal ischemia on the extracellular glutamate concentrations during secondary

ischemia in the gerbil hippocampus evaluated with intracerebral microdialysis. Neurosci.

Lett. 138:86-88.

25. Bowery, N.G., Wong, E.H.F. and Hudson, A.L. 1988. Quantitative autoradiography of $\left[{ }^{3} \mathrm{H}\right]-\mathrm{MK}-801$ binding sites in mammalian brain. Br. J. Pharmacol. 93:944-954.

26. Asai, S., Zhao, H., Takahashi, Y., Nagata, T., Kohno, T. and Ishikawa, K. 1998.

Minimal effect of brain temperature changes on glutamate release in rat following severe global brain ischemia: a dialysis electrode study. Neuroreport 9:3863-3868.

27. Zhao, H., Asai, S., Kohno, T. and Ishikawa, K. 1998. Effects of brain temperature on CBF thresholds for extracellular glutamate release and reuptake in the striatum in a rat. Neuroreport 9:3183-3188. 


\section{Figure captions}

\section{Fig. 1}

Preconditioning (PC)-induced increase in ischemic tolerance and decrease in resistance to glutamate-mediated toxicity of neurons in mixed neuron/astrocyte cultures. Figure A shows the effective time interval between the sublethal 60 min OGD (PC) and the lethal 90 min OGD for the development of PC-induced neuronal ischemic tolerance. Treatment of co-cultures with AP-5 during lethal OGD significantly protected neurons from delayed death. Photomicrographs B, and C show the sham-treated cultures exposed to glutamate (85 $\mu \mathrm{M}$ ) for $20 \mathrm{~min}(\mathrm{~B})$, and the preconditioned cultures exposed to the same concentration of glutamate for $20 \mathrm{~min}(\mathrm{C})$. Photomicrographs B1 and C1 show the cultures before exposure to glutamate, whereas B2 and C2 illustrate their state $24 \mathrm{~h}$ after the exposure, respectively. Cell nuclei were stained with bisbenzimide (Hoechst 33342) and propidium iodide (PI) (B3 and C3). Red nuclei in B3 and C3 indicate PI-positive dead neurons. Figure D shows a statistical comparison of the survival rate of neurons. The scale bar indicates $200 \mu \mathrm{m}$. Data are expressed as the mean+SD ( $\mathrm{n}=4$ different cultures). ${ }^{*} \mathrm{p}<0.05$. Abbreviations: LI, lethal OGD; PC, sublethal OGD (preconditioning). 


\section{Fig. 2}

Elevation in the extracellular concentration of glutamate (Glu) during oxygen/glucose deprivation (OGD). Photomicrographs A1, B1, and C1 show the phase-contrast images of the mixed neuron/astrocyte cultures. Figures A2-A3, B2-B3, and C2-C3 illustrate the pseudo-color representation of NADH fluorescence reflecting the extracellular concentration of glutamate (Glu) when the sham-treated cultures (A2, A3), the cultures preconditioned $24 \mathrm{~h}$ before (B2, B3), and the sham-treated cultures with DHK (200 $\mu \mathrm{M})$ treatment (C2, C3), were exposed to OGD, respectively. Figures A2, B2, C2 and A3, B3, C3 represent the fluorescence at 10 and 90 min after the onset of OGD, respectively. The fluorescent intensity increases from dark blue to red through yellow. Figure D shows a time course of the change in the NADH fluorescence ratio during OGD in the sham-treated cultures (pink), in the preconditioned cultures (orange), and in the sham-treated cultures with DHK treatment, respectively. The OGD begins at 0 min. Vertical bars indicate + SD. Neither neurons nor astrocytes were degenerated at all at $90 \mathrm{~min}$ after the start of OGD (lethal OGD). Figure E shows a statistical comparison of the relative fluorescence intensity 90 min after the onset of OGD. The scale bar indicates $200 \mu \mathrm{m}$. Data are expressed as the 
mean $+\mathrm{SD}$ ( $\mathrm{n}=4$ different cultures). $\quad * \mathrm{p}<0.05$. Abbreviations: F0, fluorescence intensity just before the onset of OGD; F, fluorescence intensity during OGD.

Fig. 3

Preconditioning (PC)-induced transient down-regulation of the expression of the astrocytic glutamate transpoter GLT-1. Western blot analysis of GLT-1 (top), GLAST (middle), and actin (bottom) in the preconditioned (24 h or $72 \mathrm{~h}$ after the PC insult) and sham-treated co-cultures (A). In each lane, $40 \mu \mathrm{g}$ of protein from different cultures was loaded. Lanes 1 and 2 show the GLT-1, GLAST and actin detected in sham-treated cultures. Lanes 3 and 4 show 24h after the PC, and lanes 5 and 6 show 72h after the PC, respectively. Figures B-D show the quantification of GLT-1, GLAST, and actin. The expression levels are normalized with the mean expression level in the sham-treated cultures. Data are expressed as the mean $+\mathrm{SD}(\mathrm{n}=4$ different cultures). $\quad * \mathrm{p}<0.05 . \quad$ An immunocytochemical analysis of the co-cultures using anti-GLT-1 antibody revealed that the level of astrocytic GLT-1was decreased in the preconditioned cultures (E2) $24 \mathrm{~h}$ after the PC as compared with that in the sham-treated cultures (E1). GLT-1-positive neurons were not detected. The scale bar indicates $200 \mu \mathrm{m}$. 


\section{Fig. 4}

Neuron-derived factors are involved in the preconditioning (PC)-induced down-regulation of glutamate transporter GLT-1. Treatment of astrocyte-enriched cultures with NCM increased the expression of GLT-1 $24 \mathrm{~h}$ after the treatment, but treatment with PC-NCM did not. Photomicrographs A1 and B1 show the phase-contrast images of the mixed neuron/astrocyte co-cultures (A1) or astrocyte-enriched cultures (B1). An immunocytochemical analysis using anti-MAP-2 (A2 and B2) and anti-GFAP antibodies (A3 and B3) indicates that the co-cultures were mixed (A2 and A3). In astrocyte-enriched cultures, few MAP-2-positive neurons were observed (B2), and almost all the cells were GFAP-positive astrocytes (B3). In addition, GLT-1-positive astrocytes were identified in neuron/astrocyte co-cultures (A4), but were scarcely detected in astrocyte-enriched cultures (B4). Figure C indicates a Western blot of GLT-1 (upper), and actin (lower) in astrocyte-enriched cultures treated with either NCM or PC-NCM. In each lane, $20 \mu \mathrm{g}$ of protein from different cultures was loaded. Lane 1 shows the GLT-1 and actin detected in non-operation (control) cultures. Lanes 2 and 3 show the GLT-1 and actin detected in cultures treated with NCM, while lanes 4 and 5 show that in cultures treated with PC-NCM, respectively. 
Figures D and E show the quantification of GLT-1 and actin. The expression levels were normalized with the mean expression level in the NCM-treated cultures. The scale bar indicates $200 \mu \mathrm{m}$. Data are expressed as the mean + SD ( $\mathrm{n}=4$ different cultures). $\quad * \mathrm{p}<0.05$. 

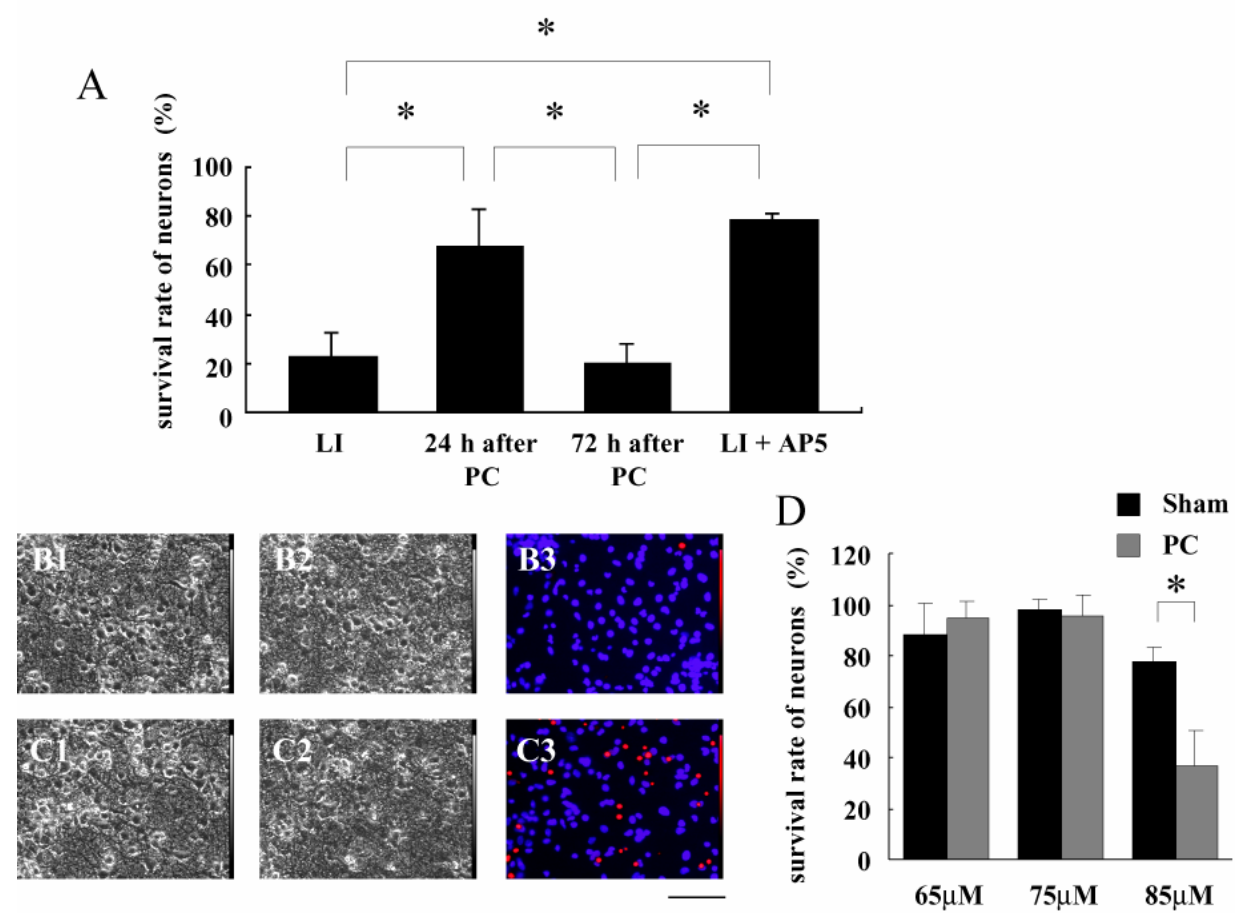

Fig. 1 

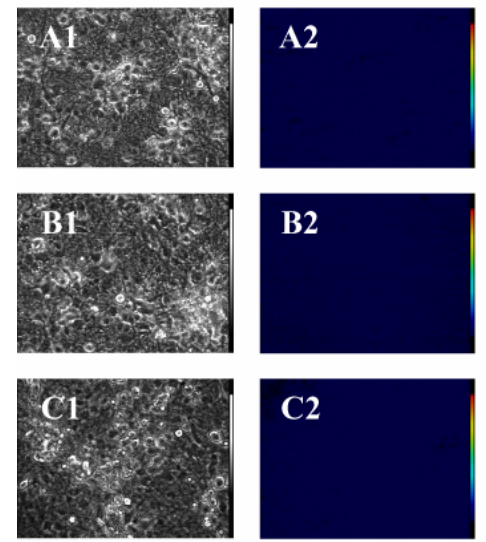

B2

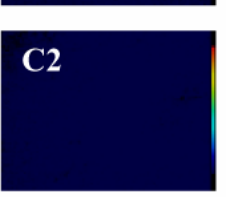

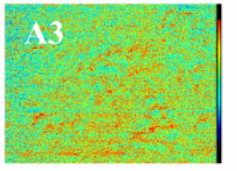
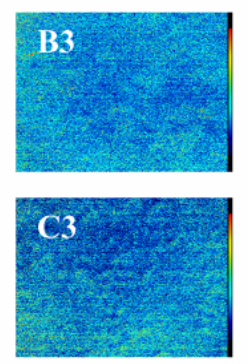

D
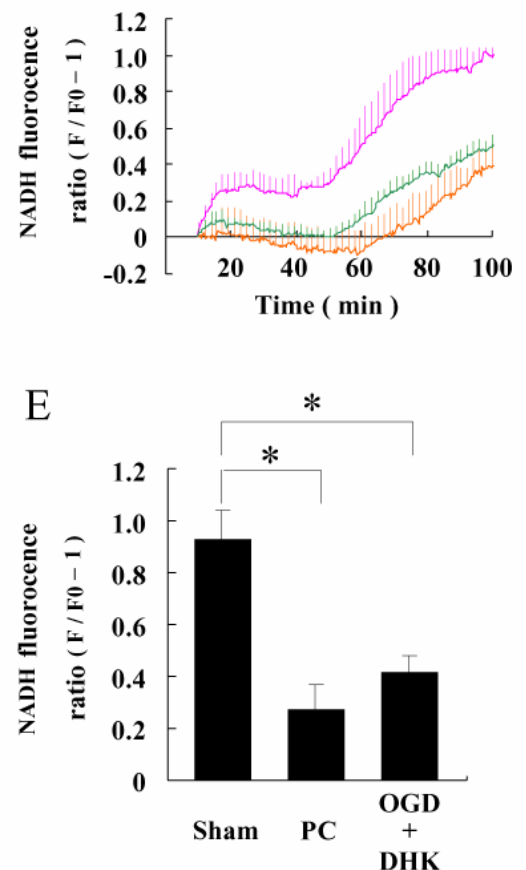

Fig. 2 

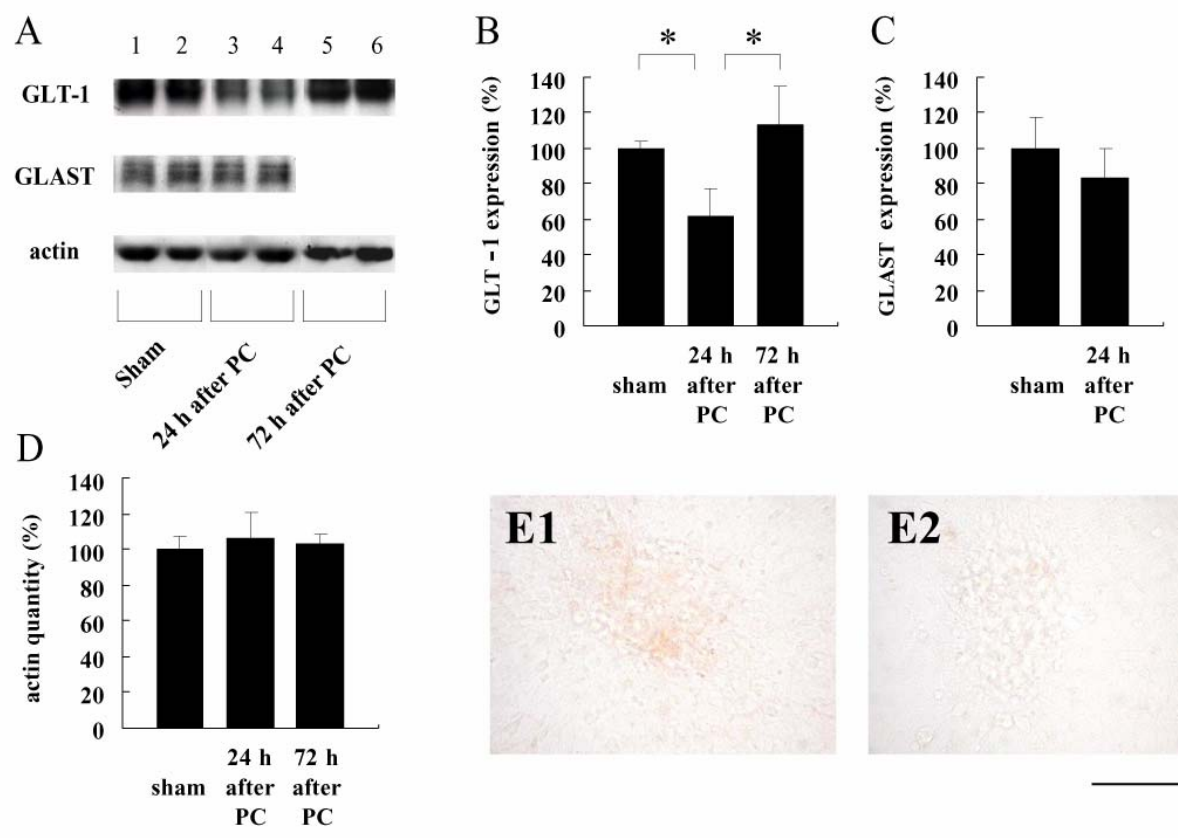

E1

E2

Fig. 3 

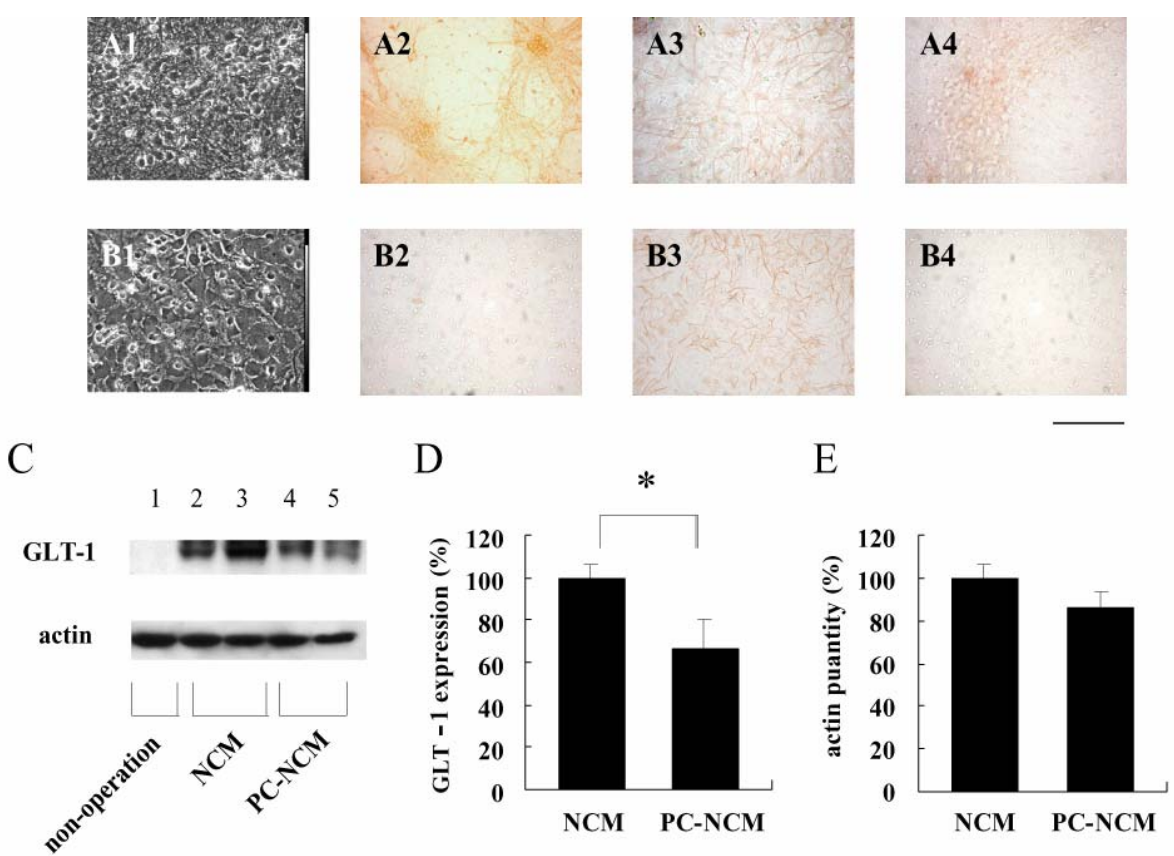

Fig. 4 


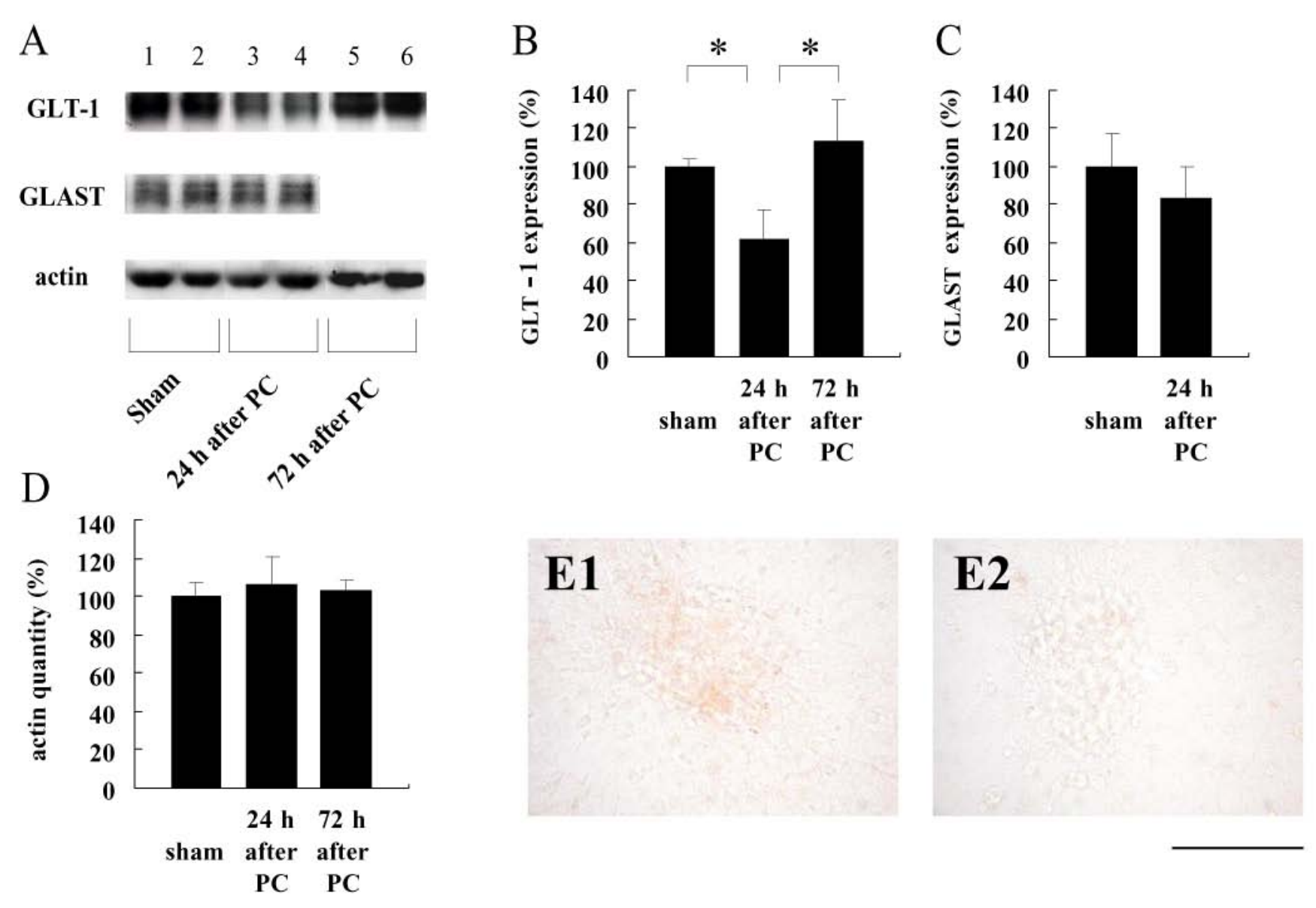



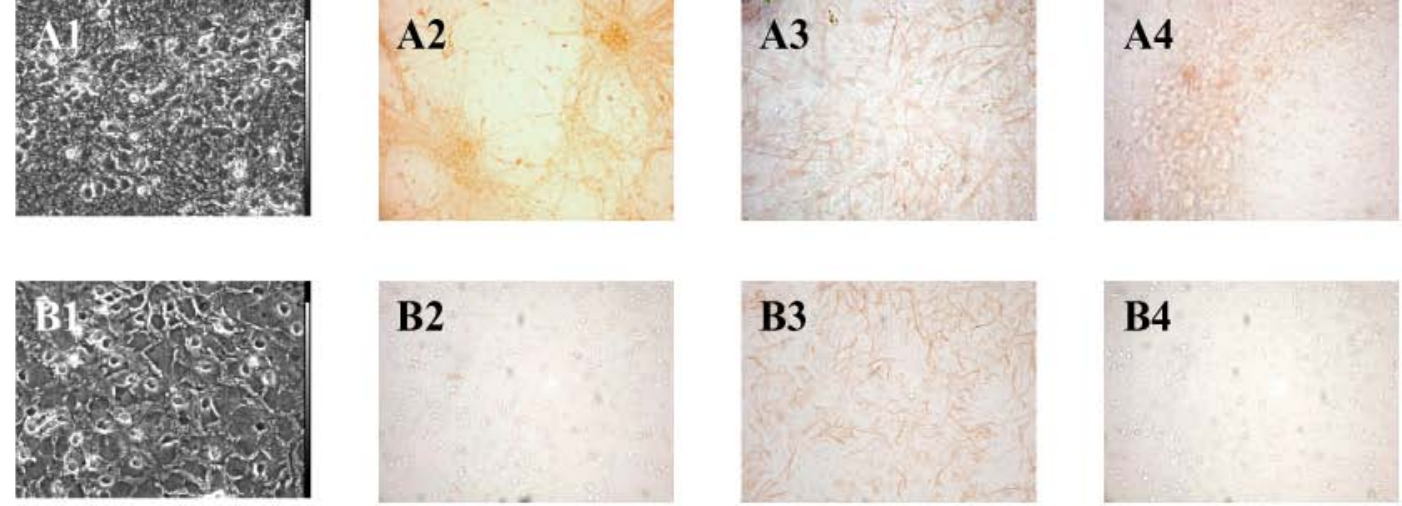

\section{B4}

D

$\begin{array}{lllll}1 & 2 & 3 & 4 & 5\end{array}$

GLT-1

actin

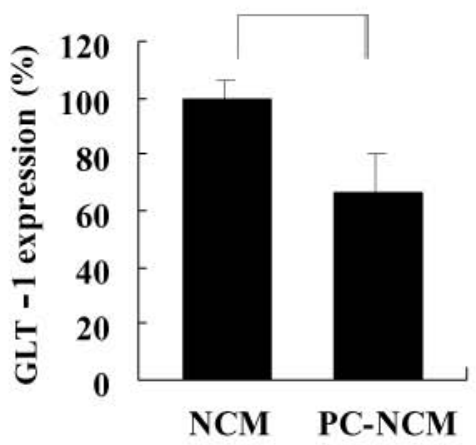

E

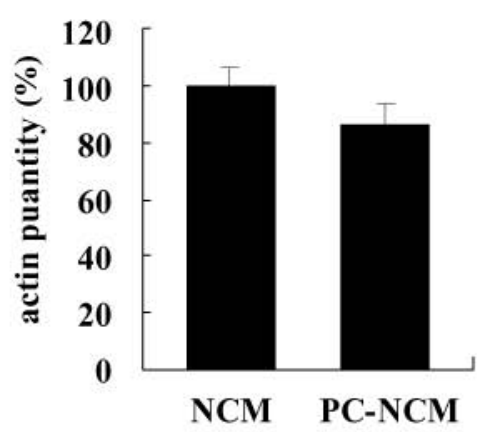

